\title{
One Adaptive Synchronization Approach for Fractional-Order Chaotic System with Fractional-Order $1<q<2$
}

\author{
Ping Zhou ${ }^{1,2}$ and Rongji Bai ${ }^{1,2}$ \\ ${ }^{1}$ Center of System Theory and Its Applications, Chongqing University of Posts and Telecommunications, Chongqing 400065, China \\ ${ }^{2}$ Key Laboratory of Network Control and Intelligent Instrument of Ministry of Education, Chongqing University of Posts and \\ Telecommunications, Chongqing 400065, China
}

Correspondence should be addressed to Ping Zhou; zhouping@cqupt.edu.cn

Received 19 May 2014; Accepted 11 August 2014; Published 27 August 2014

Academic Editor: Jianquan Lu

Copyright (C) 2014 P. Zhou and R. Bai. This is an open access article distributed under the Creative Commons Attribution License, which permits unrestricted use, distribution, and reproduction in any medium, provided the original work is properly cited.

Based on a new stability result of equilibrium point in nonlinear fractional-order systems for fractional-order lying in $1<q<2$, one adaptive synchronization approach is established. The adaptive synchronization for the fractional-order Lorenz chaotic system with fractional-order $1<q<2$ is considered. Numerical simulations show the validity and feasibility of the proposed scheme.

\section{Introduction}

Fractional-order differential equations can be more accurately described in the real-world physical systems [1-3]. Many fractional-order systems create chaotic attractor. Many fractional-order chaotic attractors have been reported in recent years, for example, the fractional-order Lorenz chaotic attractor $[1,4,5]$, the fractional-order Chen chaotic attractor [5], the fractional-order Lu chaotic attractor $[2,4]$, the fractional-order Chua chaotic attractor [5], the fractionalorder Duffing chaotic attractor [6], the fractional-order Rössler chaotic attractor $[7,8]$, and so on. On the other hand, synchronization of chaotic systems has been given more attention [2,9-13]. This is due to its applications in the field of engineering and science. Over the last two decades, many scholars have proposed various synchronization schemes. It is well known that many chaotic systems in practical situations are usually with fully or partially unknown parameters. In order to estimate the unknown parameters, a synchronization scheme named adaptive synchronization has been proposed. Now, the adaptive synchronization [13-16] has attracted more and more attention. This is due to its effectiveness in many practical chaos applications.
However, many adaptive synchronization approaches on fractional-order chaotic systems reported previously [2, 913] were considered the fractional-order lying in $0<q<1$. To the best of our knowledge, there are a few results about adaptive synchronization on fractional-order chaotic systems with fractional-order $1<q<2$. In fact, there are many fractional-order systems with fractional-order $1<q<2$ in real-world physical systems, for example, the fractional diffusion-wave equation [17], the fractional telegraph equation [18], the time fractional heat conduction equation [19], and so forth. So, an interesting question is how to realize the adaptive synchronization for fractional-order lying in $1<q<$ 2 ? This question is of practical importance as well as academic significance. In this paper, a positive answer is given for the above question.

Inspired by the above-mentioned discussion, one adaptive synchronization approach for a class of fractional-order chaotic system with $1<q<2$ is established. This approach is based on a new stability result of equilibrium point in nonlinear fractional-order systems for fractional-order lying in $1<q<2$ [1]. The adaptive synchronization for the fractional-order Lorenz chaotic system with fractional-order $1<q<2$ is considered. Numerical simulations show the validity and feasibility of the proposed scheme. 


\section{Preliminaries and Main Results}

In our paper, the $q$ th Caputo derivative for function $g(t)$ is shown as

$$
D^{q} g(t)=\frac{1}{\Gamma(l-q)} \int_{0}^{t} \frac{g^{(l)}(\tau)}{(t-\tau)^{q+1-l}} d \tau, \quad l-1<q<l,
$$

where $D^{q}$ denote the Caputo derivative, $l$ is the smallest integer larger than $q, g^{(l)}(t)$ is the $l$ th derivative in the usual sense, and $\Gamma$ is the gamma function.

Now, consider the following fractional-order chaotic system:

$$
D^{q} x=f(x)=M x+n(x),
$$

where fractional-order $1<q<2, x \in R^{n \times 1}$, and $f(x) \in R^{n \times 1}$. $M \in R^{n \times n}$ is a constant matrix. $n(x) \in R^{n \times 1}$ is the nonlinear part of system (2).

The system (2) can be rewritten as follows:

$$
D^{q} x=L\left(\begin{array}{c}
x \\
\sigma_{0}
\end{array}\right)+n\left(x, \sigma_{0}\right),
$$

where $\sigma_{0} \in R$ is the system parameter. $n\left(x, \sigma_{0}\right) \in R^{n \times 1}$ is the nonlinear part, and all the terms with system parameter $\sigma_{0}$ are contained in $n\left(x, \sigma_{0}\right) . L \in R^{n \times(n+1)}$ is a constant matrix, and matrix element $L_{i, n+1}=0(i=1,2, \ldots, n)$.

In this paper, we focus on a class of fractional-order chaotic system in which the equation $n\left(y, \sigma_{0}\right)-n\left(x, \sigma_{0}\right)=$ $n_{l p}(x)(y-x)+n_{n p}(y-x, x)$ holds. Here, variable $y \in R^{n \times 1}$ is real number. Vector $n_{l p}(x)(y-x)$ and vector $n_{n p}(y-$ $x, x)$ are the linear part and nonlinear part with respect to $(y-x)$, respectively. In fact, the nonlinear term $n\left(x, \sigma_{0}\right)$ in many fractional-order chaotic systems meet this equation, for example, the fractional-order Lorenz chaotic system, fractional-order Chen chaotic system, fractional-order Lu chaotic system, fractional-order Rössler chaotic system, the fractional-order Chua's chaotic system and its modified chaotic system, the fractional-order Duffing chaotic system, the fractional-order Arneodo chaotic system, the fractionalorder Sprott chaotic system, and so forth.

Next, the adaptive synchronization for fractional-order chaotic system (3) is proposed. Select system (3) as drive system; the response systems with parameter update law are shown as follows:

$$
\begin{gathered}
D^{q} y=L\left(\begin{array}{l}
y \\
\sigma
\end{array}\right)+n(y, \sigma)+u(x, y, \sigma), \\
D^{q} \sigma=\Omega e,
\end{gathered}
$$

where $y \in R^{n \times 1}$ is state vector, $u(x, y, \sigma) \in R^{n \times 1}$ is a controller, $\Omega \in R^{1 \times(n+1)}$ is real constant matrix, and parameter $\sigma$ is unknown in response system (4). The true value of the "unknown" parameter $\sigma$ is selected as $\sigma_{0}$. The parameter update law is $D^{q} \sigma=\Omega e$. The adaptive synchronization errors are $e=\left(e_{1}, \ldots, e_{n}, e_{n+1}\right)^{\mathrm{T}} \in R^{(n+1) \times 1}, e_{i}=\left(y_{i}-x_{i}\right) \in R(i=$ $1,2, \ldots, n)$, and $e_{n+1}=e_{\sigma}=\left(\sigma-\sigma_{0}\right) \in R$.
Lemma 1 (see [1]). For the nonlinear part $n(x)$ of systems (2), if

(i) $\left.n(x)\right|_{x=0}=0, \lim _{x \rightarrow 0}(\|n(x)\| /\|x\|)=0$;

(ii) $\operatorname{Re}[\lambda(M)]<0,-\max [\operatorname{Re} \lambda(M)]>[\Gamma(q)]^{1 / q}$.

Then, the zero solution of fractional-order chaotic system (2) is asymptotically stable.

Based on this lemma, the following main results are given.

Theorem 2. If the controller is selected as

$$
u(x, y, \sigma)=\left[F-n_{l p}(x)\right] e
$$

and the following conditions are satisfied:

(i) $\left.\left(\begin{array}{c}n_{n p}(e, x) \\ 0\end{array}\right)\right|_{e=0}=0, \lim _{e \rightarrow 0}\left(\left\|\left(\begin{array}{c}n_{n p}(e, x) \\ 0\end{array}\right)\right\| /\|e\|\right)=0$ for any $x$,

(ii) $\operatorname{Re}\left[\lambda\left(\begin{array}{c}L+F \\ \Omega\end{array}\right)\right]<0,-\max \left[\operatorname{Re} \lambda\left(\begin{array}{c}L+F \\ \Omega\end{array}\right)\right]>[\Gamma(q)]^{1 / q}$,

then the adaptive synchronization between fractional-order chaotic system (3) and fractional-order system (4) can be arrived, where $n(y, \sigma)-n\left(x, \sigma_{0}\right)=n_{l p}(x) e+n_{n p}(e, x), n_{l p}(x) \epsilon$ $R^{n \times(n+1)}$, and $n_{n p}(e, x) \in R^{n \times 1} \cdot F \in R^{n \times(n+1)}$ is a suitable constant matrix.

Proof. The error system between systems (4) and (3) can be shown as

$$
\begin{gathered}
D^{q}(y-x)=L\left(\left(\begin{array}{c}
y \\
\sigma
\end{array}\right)-\left(\begin{array}{c}
x \\
\sigma_{0}
\end{array}\right)\right)+n(y, \sigma) \\
-n\left(x, \sigma_{0}\right)+u(x, y, \sigma) . \\
D^{q} \sigma=\Omega e .
\end{gathered}
$$

Due to $e=\left(e_{1}, \ldots, e_{n}, e_{n+1}\right)^{\mathbf{T}}, e_{i}=y_{i}-x_{i}(i=1,2, \ldots, n)$, and $e_{n+1}=e_{\sigma}=\left(\sigma-\sigma_{0}\right)$, system (6) can be rewritten as

$$
\begin{gathered}
D^{q}(y-x)=L e+n(y, \sigma)-n\left(x, \sigma_{0}\right)+u(x, y, \sigma), \\
D^{q} \sigma=\Omega e .
\end{gathered}
$$

Using $n(y, \sigma)-n\left(x, \sigma_{0}\right)=n_{l p}(x) e+n_{n p}(e, x), D^{q} \sigma_{0}=0$, and $D^{q} \sigma=D^{q}\left(\sigma-\sigma_{0}\right)=D^{q} e_{\sigma}$, error system (7) can be changed as

$$
\begin{gathered}
D^{q}(y-x)=L e+n_{l p}(x) e+n_{n p}(e, x)+u(x, y, \sigma), \\
D^{q} e_{\sigma}=\Omega e .
\end{gathered}
$$

Since $u(x, y, \sigma)=\left[F-n_{l p}(x)\right] e$ and $e=\left(e_{1}, \ldots, e_{n}, e_{n+1}\right)^{\mathrm{T}}$, therefore, (8) can be changed as

$$
D^{q} e=\left(\begin{array}{c}
L+F \\
\Omega
\end{array}\right) e+\left(\begin{array}{c}
n_{n p}(e, x) \\
0
\end{array}\right)
$$



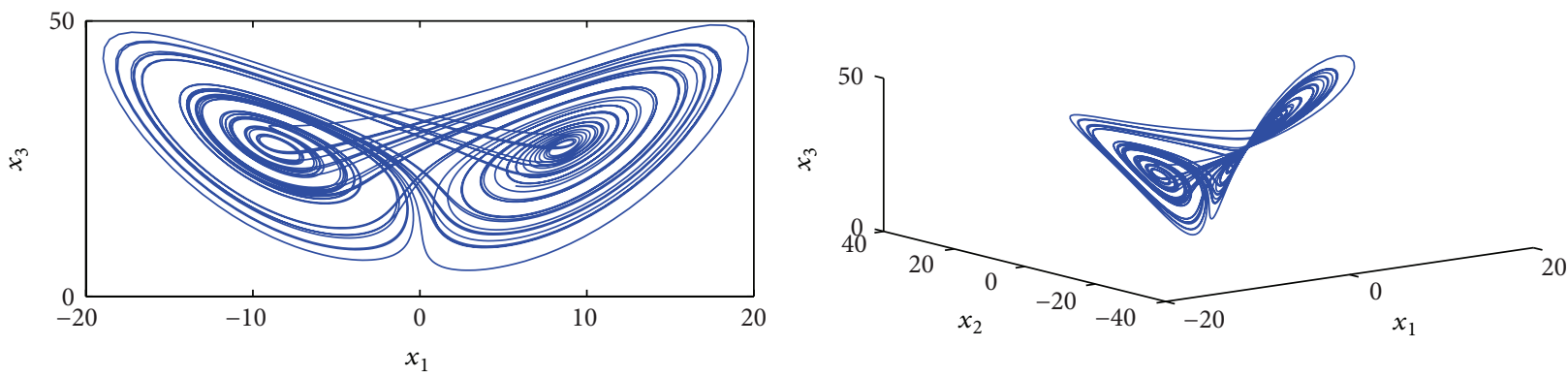

FIgURE 1: The attractor of fractional-order Lorenz system (12) for $q=1.05$.

Due to $\left.\left(\begin{array}{c}n_{n p}(e, x) \\ 0\end{array}\right)\right|_{e=0}=0, \lim _{e \rightarrow 0}\left(\left\|\left(\begin{array}{c}n_{n p}(e, x) \\ 0\end{array}\right)\right\| /\|e\|\right)=$ 0 for any $x, \operatorname{Re}\left[\lambda\left(\begin{array}{c}L+F \\ \Omega\end{array}\right)\right]<0$, and $-\max \left[\operatorname{Re} \lambda\left(\begin{array}{c}L+F \\ \Omega\end{array}\right)\right]>$ $[\Gamma(q)]^{1 / q}$. According to the above-mentioned lemma, the zero solution of fractional-order system (9) is asymptotically stable. So, the following result holds:

$$
\lim _{t \rightarrow+\infty}\|e\|=0
$$

It implies the following:

$$
\lim _{t \rightarrow+\infty}\|y-x\|=0, \quad \lim _{t \rightarrow+\infty}\left(\sigma-\sigma_{0}\right)=0 .
$$

Therefore, the adaptive synchronization between fractional-order chaotic system (3) and fractional-order system (4) can be arrived. The proof is completed.

\section{Illustrative Example}

In this section, to show the effectiveness of the adaptive synchronization approach in this paper, the adaptive synchronization for the fractional-order Lorenz chaotic system [4] with fractional-order $1<q<2$ is considered. Numerical simulations show the validity and feasibility of the proposed scheme.

The fractional-order Lorenz system is described by

$$
\left(\begin{array}{c}
D^{q} x_{1} \\
D^{q} x_{2} \\
D^{q} x_{3}
\end{array}\right)=\left(\begin{array}{c}
a_{0}\left(x_{2}-x_{1}\right) \\
b_{0} x_{1}-x_{2}-x_{1} x_{3} \\
x_{1} x_{2}-c_{0} x_{3}
\end{array}\right),
$$

where $a_{0}, b_{0}$, and $c_{0}$ are system parameters. Let $a_{0}=10, b_{0}=$ $28, c_{0}=8 / 3$, and $q=1.05$; the system (12) creates chaotic attractor. The chaotic attractor is shown in Figure 1.

Case 1 (parameter $a$ is unknown in response system). Now, assume the system parameter $a$ in in response system is the unknown parameter. The true value of the "unknown" parameter $a$ is selected as $a_{0}$.
The fractional-order Lorenz system (12) can be rewritten as

$$
\begin{aligned}
\left(\begin{array}{c}
D^{q} x_{1} \\
D^{q} x_{2} \\
D^{q} x_{3}
\end{array}\right)= & \left(\begin{array}{cccc}
0 & 0 & 0 & 0 \\
28 & -1 & 0 & 0 \\
0 & 0 & -\frac{8}{3} & 0
\end{array}\right)\left(\begin{array}{l}
x_{1} \\
x_{2} \\
x_{3} \\
a_{0}
\end{array}\right) \\
& +\left(\begin{array}{c}
a_{0}\left(x_{2}-x_{1}\right) \\
-x_{1} x_{3} \\
x_{1} x_{2}
\end{array}\right),
\end{aligned}
$$

where $a_{0}=10$.

So

$$
L=\left(\begin{array}{cccc}
0 & 0 & 0 & 0 \\
28 & -1 & 0 & 0 \\
0 & 0 & -\frac{8}{3} & 0
\end{array}\right)
$$

According to Section 2, the response system with unknown parameter $a$ can be given as

$$
\begin{aligned}
\left(\begin{array}{l}
D^{q} y_{1} \\
D^{q} y_{2} \\
D^{q} y_{3}
\end{array}\right)= & \left(\begin{array}{cccc}
0 & 0 & 0 & 0 \\
28 & -1 & 0 & 0 \\
0 & 0 & -\frac{8}{3} & 0
\end{array}\right)\left(\begin{array}{c}
y_{1} \\
y_{2} \\
y_{3} \\
a
\end{array}\right) \\
& +\left(\begin{array}{c}
a\left(y_{2}-y_{1}\right) \\
-y_{1} y_{3} \\
y_{1} y_{2}
\end{array}\right)+\left(F-n_{l p}(x)\right)\left(\begin{array}{c}
e_{1} \\
e_{2} \\
e_{3} \\
e_{a}
\end{array}\right)
\end{aligned}
$$

and the parameter update law is

$$
D^{q} a=\Omega\left(\begin{array}{llll}
e_{1} & e_{2} & e_{3} & e_{a}
\end{array}\right)^{\mathrm{T}} .
$$

It is easy to obtain the following:

$$
\begin{gathered}
\left(\begin{array}{c}
a\left(y_{2}-y_{1}\right) \\
-y_{1} y_{3} \\
y_{1} y_{2}
\end{array}\right)-\left(\begin{array}{c}
a_{0}\left(x_{2}-x_{1}\right) \\
-x_{1} x_{3} \\
x_{1} x_{2}
\end{array}\right) \\
=\left(\begin{array}{cccc}
-a_{0} & a_{0} & 0 & x_{2}-x_{1} \\
-x_{3} & 0 & -x_{1} & 0 \\
x_{2} & x_{1} & 0 & 0
\end{array}\right)\left(\begin{array}{l}
e_{1} \\
e_{2} \\
e_{3} \\
e_{a}
\end{array}\right) \\
+\left(\begin{array}{c}
e_{a}\left(e_{2}-e_{1}\right) \\
-e_{1} e_{3} \\
e_{1} e_{2}
\end{array}\right) .
\end{gathered}
$$


So

$$
\begin{gathered}
n_{l p}(x)=\left(\begin{array}{cccc}
-a_{0} & a_{0} & 0 & x_{2}-x_{1} \\
-x_{3} & 0 & -x_{1} & 0 \\
x_{2} & x_{1} & 0 & 0
\end{array}\right), \\
n_{n p}(e, x)=\left(\begin{array}{c}
e_{a}\left(e_{2}-e_{1}\right) \\
-e_{1} e_{3} \\
e_{1} e_{2}
\end{array}\right) .
\end{gathered}
$$

Now, it is easy to verify the following:

$$
\begin{aligned}
& \frac{\left\|\left(\begin{array}{c}
n_{n p}(e, x) \\
0
\end{array}\right)\right\|}{\|e\|} \\
& =\sqrt{\frac{e_{a}^{2}\left(e_{2}-e_{1}\right)^{2}+\left(e_{1} e_{3}\right)^{2}+\left(e_{1} e_{2}\right)^{2}}{e_{1}^{2}+e_{2}^{2}+e_{3}^{2}+e_{a}^{2}}} \\
& \leq \sqrt{\frac{e_{a}^{2}\left(\left|e_{2}\right|+\left|e_{1}\right|\right)^{2}+\left(e_{1} e_{3}\right)^{2}+\left(e_{1} e_{2}\right)^{2}}{e_{1}^{2}+e_{2}^{2}+e_{3}^{2}+e_{a}^{2}}} \\
& =\sqrt{\frac{e_{a}^{2}\left(\left|e_{2}\right|+\left|e_{1}\right|\right)^{2}}{e_{1}^{2}+e_{2}^{2}+e_{3}^{2}+e_{a}^{2}}+\frac{\left(e_{1} e_{3}\right)^{2}+\left(e_{1} e_{2}\right)^{2}}{e_{1}^{2}+e_{2}^{2}+e_{3}^{2}+e_{a}^{2}}} \\
& \leq \sqrt{\frac{e_{a}^{2}\left(\left|e_{2}\right|+\left|e_{1}\right|\right)^{2}}{e_{a}^{2}}+\frac{\left(e_{1} e_{3}\right)^{2}+\left(e_{1} e_{2}\right)^{2}}{e_{1}^{2}}} \\
& =\sqrt{\left(\left|e_{2}\right|+\left|e_{1}\right|\right)^{2}+\left(e_{2}\right)^{2}+\left(e_{3}\right)^{2}}, \\
& \lim _{e \rightarrow 0} \frac{\left\|\left(\begin{array}{c}
n_{n p}(e, x) \\
0
\end{array}\right)\right\|}{\|e\|} \\
& \leq \lim _{e \rightarrow 0} \sqrt{\left(\left|e_{2}\right|+\left|e_{1}\right|\right)^{2}+\left(e_{2}\right)^{2}+\left(e_{3}\right)^{2}}=0, \\
& \left.\left(\begin{array}{c}
n_{n p}(e, x) \\
0
\end{array}\right)\right|_{e=0}=0 .
\end{aligned}
$$

Therefore, the first condition in the above-mentioned theorem holds.

Now, choose suitable real constant matrix $\Omega \in R^{1 \times n}$ and $F \in R^{n \times(n+1)}$ such that

$$
\begin{gathered}
\operatorname{Re}\left[\lambda\left(\begin{array}{c}
L+F \\
\Omega
\end{array}\right)\right]<0, \\
-\max \left[\operatorname{Re} \lambda\left(\begin{array}{c}
L+F \\
\Omega
\end{array}\right)\right]>[\Gamma(q)]^{1 / q} .
\end{gathered}
$$

So the second condition in the above-mentioned theorem holds. According to the theorem in Section 2, the adaptive synchronization between drive system (13) and response system (15) with parameter update law (16) can be achieved.

For example, let $F=\left(\begin{array}{cccc}-1 & 0 & 0 & 0 \\ -2 & 0 & 0 & 0 \\ 0 & 0 & 0 & 0\end{array}\right)$ and $\Omega=\left(\begin{array}{llll}0 & 0 & 0 & -1\end{array}\right)$. So $\left(\begin{array}{c}L+F \\ \Omega\end{array}\right)=\left(\begin{array}{cccc}-1 & 0 & 0 & 0 \\ 0 & -1 & 0 & 0 \\ 0 & 0 & -8 / 3 & 0 \\ 0 & 0 & 0 & -1\end{array}\right)$. Therefore, $\lambda_{i}=-1(i=1,2,3)$,
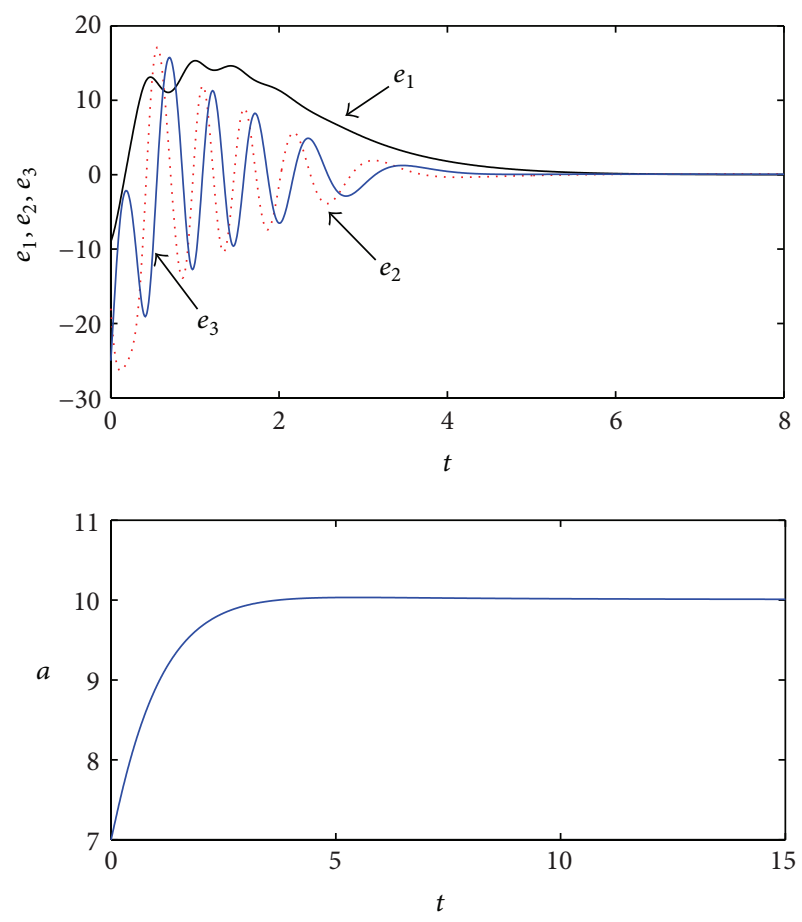

FIGURE 2: Simulation results of the adaptive synchronization for the fractional-order Lorenz chaotic system.

$\lambda_{4}=-8 / 3$, and $-\max \left[\operatorname{Re} \lambda\left(\begin{array}{c}L+F \\ \Omega\end{array}\right)\right]=1>[\Gamma(q)]^{1 / q}=0.9722$, respectively. Simulation results are shown in Figure 2. Here, $a(0)=7$, and all the initial conditions in this paper are $\left(x_{10}, x_{20}, x_{30}\right)=(10,20,30)$, and $\left(y_{10}, y_{20}, y_{30}\right)=(1,2,5)$, respectively.

Case 2 (parameter $b$ is unknown in response system). Now, assume that the system parameter $b$ in response system is the unknown parameter. The true value of the "unknown" parameter $b$ is selected as $b_{0}$.

The fractional-order Lorenz system (12) can be rewritten as

$$
\left(\begin{array}{l}
D^{q} x_{1} \\
D^{q} x_{2} \\
D^{q} x_{3}
\end{array}\right)=\left(\begin{array}{cccc}
-10 & 10 & 0 & 0 \\
0 & -1 & 0 & 0 \\
0 & 0 & -\frac{8}{3} & 0
\end{array}\right)\left(\begin{array}{c}
x_{1} \\
x_{2} \\
x_{3} \\
b_{0}
\end{array}\right)+\left(\begin{array}{c}
0 \\
b_{0} x_{1}-x_{1} x_{3} \\
x_{1} x_{2}
\end{array}\right)
$$

where $b_{0}=28$.

So

$$
L=\left(\begin{array}{cccc}
-10 & 10 & 0 & 0 \\
0 & -1 & 0 & 0 \\
0 & 0 & -\frac{8}{3} & 0
\end{array}\right)
$$


According to Section 2, the response system with unknown parameter $b$ can be given as

$$
\begin{aligned}
\left(\begin{array}{l}
D^{q_{2}} y_{1} \\
D^{q_{2}} y_{2} \\
D^{q_{2}} y_{3}
\end{array}\right)= & \left(\begin{array}{cccc}
-10 & 10 & 0 & 0 \\
0 & -1 & 0 & 0 \\
0 & 0 & -\frac{8}{3} & 0
\end{array}\right)\left(\begin{array}{c}
y_{1} \\
y_{2} \\
y_{3} \\
b
\end{array}\right) \\
& +\left(\begin{array}{c}
0 \\
b y_{1}-y_{1} y_{3} \\
y_{1} y_{2}
\end{array}\right)+\left(F-n_{l p}(x)\right)\left(\begin{array}{l}
e_{1} \\
e_{2} \\
e_{3} \\
e_{b}
\end{array}\right)
\end{aligned}
$$

and the parameter update law is

$$
D^{q} b=\Omega\left(\begin{array}{llll}
e_{1} & e_{2} & e_{3} & e_{b}
\end{array}\right)^{\mathrm{T}}
$$

It is easy to obtain the following:

$$
\begin{gathered}
\left(\begin{array}{c}
0 \\
b y_{1}-y_{1} y_{3} \\
y_{1} y_{2}
\end{array}\right)-\left(\begin{array}{c}
0 \\
b_{0} x_{1}-x_{1} x_{3} \\
x_{1} x_{2}
\end{array}\right) \\
=\left(\begin{array}{cccc}
0 & 0 & 0 & 0 \\
b_{0}-x_{3} & 0 & -x_{1} & 0 \\
x_{2} & x_{1} & 0 & 0
\end{array}\right)\left(\begin{array}{l}
e_{1} \\
e_{2} \\
e_{3} \\
e_{b}
\end{array}\right) \\
+\left(\begin{array}{c}
0 \\
-e_{1} e_{3}+e_{b} e_{1} \\
e_{1} e_{2}
\end{array}\right)
\end{gathered}
$$

So

$$
\begin{gathered}
n_{l p}(x)=\left(\begin{array}{cccc}
0 & 0 & 0 & 0 \\
b_{0}-x_{3} & 0 & -x_{1} & 0 \\
x_{2} & x_{1} & 0 & 0
\end{array}\right), \\
n_{n p}(e, x)=\left(\begin{array}{c}
0 \\
-e_{1} e_{3}+e_{b} e_{1} \\
e_{1} e_{2}
\end{array}\right) .
\end{gathered}
$$

Now, it is easy to verify the following:

$$
\begin{aligned}
& \frac{\left\|\left(\begin{array}{c}
n_{n p}(e, x) \\
0
\end{array}\right)\right\|}{\|e\|} \\
& \quad=\sqrt{\frac{\left(-e_{1} e_{3}+e_{b} e_{1}\right)^{2}+\left(e_{1} e_{2}\right)^{2}}{e_{1}^{2}+e_{2}^{2}+e_{3}^{2}+e_{b}^{2}}} \\
& \quad \leq \sqrt{\frac{\left(\left|e_{3}\right|+\left|e_{b}\right|\right)^{2} e_{1}^{2}+\left(e_{1} e_{2}\right)^{2}}{e_{1}^{2}+e_{2}^{2}+e_{3}^{2}+e_{b}^{2}}} \\
& \quad \leq \sqrt{\frac{\left(\left|e_{3}\right|+\left|e_{b}\right|\right)^{2} e_{1}^{2}+\left(e_{1} e_{2}\right)^{2}}{e_{1}^{2}}} \\
& =\sqrt{\left(\left|e_{3}\right|+\left|e_{b}\right|\right)^{2}+\left(e_{2}\right)^{2}}
\end{aligned}
$$
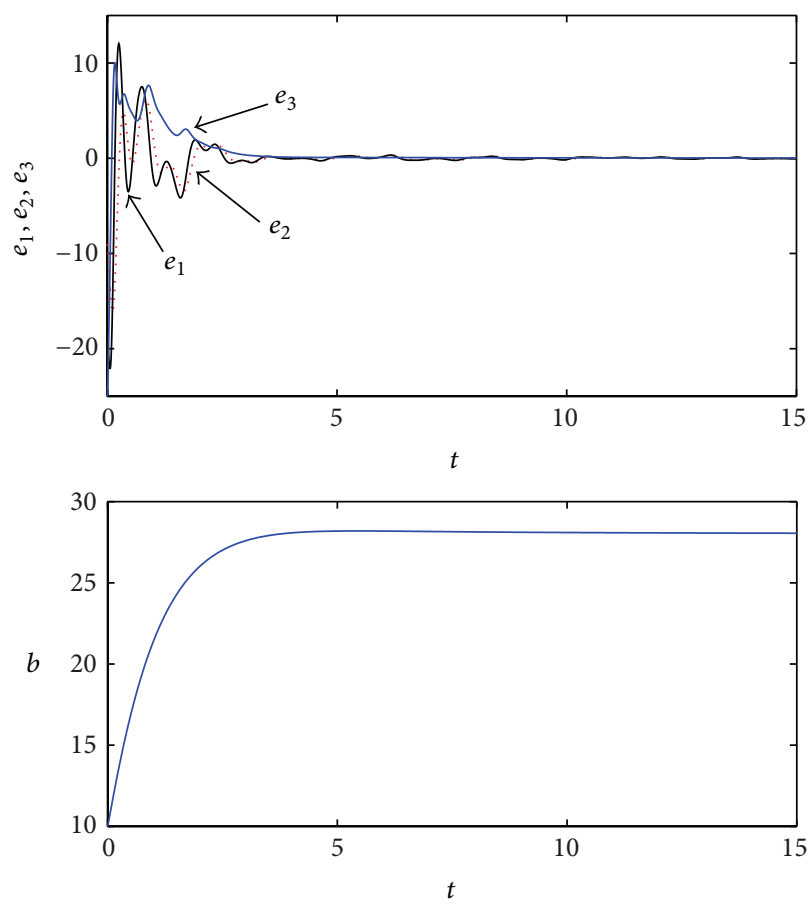

FIGURE 3: Simulation results of the adaptive synchronization for the fractional-order Lorenz chaotic system.

$$
\begin{gathered}
\lim _{e \rightarrow 0} \frac{\left\|\left(\begin{array}{c}
n_{n p}(e, x) \\
0
\end{array}\right)\right\|}{\|e\|} \leq \lim _{e \rightarrow 0} \sqrt{\left(\left|e_{3}\right|+\left|e_{b}\right|\right)^{2}+\left(e_{2}\right)^{2}}=0 \\
\left.\left(\begin{array}{c}
n_{n p}(e, x) \\
0
\end{array}\right)\right|_{e=0}=0 .
\end{gathered}
$$

Therefore, the first condition in the above-mentioned theorem holds.

Now, choose suitable real constant matrix $\Omega \in R^{1 \times n}$ and $F \in R^{n \times(n+1)}$ such that

$$
\operatorname{Re}\left[\lambda\left(\begin{array}{c}
L+F \\
\Omega
\end{array}\right)\right]<0,
$$

$$
-\max \left[\operatorname{Re} \lambda\left(\begin{array}{c}
L+F \\
\Omega
\end{array}\right)\right]>[\Gamma(q)]^{1 / q} .
$$

So the second condition in the above-mentioned theorem holds. According to the theorem in Section 2, the adaptive synchronization between drive system (21) and response system (23) with parameter update law (24) can be achieved.

For example, let $F=\left(\begin{array}{cccc}0 & 0 & 0 & 0 \\ -10 & 0 & 0 & 0 \\ 0 & 0 & 0 & 0\end{array}\right)$ and $\Omega=\left(\begin{array}{llll}0 & 0 & 0 & -1\end{array}\right)$. So $\left(\begin{array}{c}L+F \\ \Omega\end{array}\right)=\left(\begin{array}{cccc}-10 & 10 & 0 & 0 \\ -10 & -1 & 0 & 0 \\ 0 & 0 & -8 / 3 & 0 \\ 0 & 0 & 0 & -1\end{array}\right)$. Therefore, $\lambda_{ \pm}=-5.5 \pm 8.9303 j$, $\lambda_{3}=-8 / 3, \lambda_{4}=-1$, and $-\max \left[\operatorname{Re} \lambda\left(\begin{array}{c}L+F \\ \Omega\end{array}\right)\right]=1>$ $[\Gamma(q)]^{1 / q}=0.9722$, respectively. Simulation results are shown in Figure 3. Here, $b(0)=10$.

Case 3 (parameter $c$ is unknown in response system). Now, assume that the system parameter $c$ in response system is 
the unknown parameter. The true value of the "unknown" parameter $c$ is selected as $c_{0}$. as

The fractional-order Lorenz system (12) can be rewritten

So

$$
L=\left(\begin{array}{cccc}
-10 & 10 & 0 & 0 \\
28 & -1 & 0 & 0 \\
0 & 0 & 0 & 0
\end{array}\right)
$$

According to Section 2, the response system is given as

$$
\begin{aligned}
\left(\begin{array}{l}
D^{q} y_{1} \\
D^{q} y_{2} \\
D^{q} y_{3}
\end{array}\right)= & \left(\begin{array}{cccc}
-10 & 10 & 0 & 0 \\
28 & -1 & 0 & 0 \\
0 & 0 & 0 & 0
\end{array}\right)\left(\begin{array}{c}
y_{1} \\
y_{2} \\
y_{3} \\
c
\end{array}\right) \\
& +\left(\begin{array}{c}
0 \\
-y_{1} y_{3} \\
y_{1} y_{2}-c y_{3}
\end{array}\right)+\left(F-n_{l p}(x)\right)\left(\begin{array}{l}
e_{1} \\
e_{2} \\
e_{3} \\
e_{c}
\end{array}\right)
\end{aligned}
$$

and the parameter update law is

$$
D^{q} c=\Omega\left(\begin{array}{llll}
e_{1} & e_{2} & e_{3} & e_{c}
\end{array}\right)^{\mathrm{T}}
$$

It is easy to obtain the following:

$$
\begin{gathered}
\left(\begin{array}{c}
0 \\
-y_{1} y_{3} \\
y_{1} y_{2}-c y_{3}
\end{array}\right)-\left(\begin{array}{c}
0 \\
-x_{1} x_{3} \\
x_{1} x_{2}-c_{0} x_{3}
\end{array}\right) \\
=\left(\begin{array}{cccc}
0 & 0 & 0 & 0 \\
-x_{3} & 0 & -x_{1} & 0 \\
x_{2} & x_{1} & -c_{0} & -x_{3}
\end{array}\right)\left(\begin{array}{l}
e_{1} \\
e_{2} \\
e_{3} \\
e_{c}
\end{array}\right) \\
+\left(\begin{array}{c}
0 \\
-e_{1} e_{3} \\
e_{1} e_{2}-e_{c} e_{3}
\end{array}\right) .
\end{gathered}
$$

So

$$
\begin{gathered}
n_{l p}(x)=\left(\begin{array}{cccc}
0 & 0 & 0 & 0 \\
-x_{3} & 0 & -x_{1} & 0 \\
x_{2} & x_{1} & -c_{0} & -x_{3}
\end{array}\right), \\
n_{n p}(e, x)=\left(\begin{array}{c}
0 \\
-e_{1} e_{3} \\
e_{1} e_{2}-e_{c} e_{3}
\end{array}\right) .
\end{gathered}
$$

Now, it is easy to verify the following:

$$
\begin{aligned}
& \frac{\left\|\left(\begin{array}{c}
n_{n p}(e, x) \\
0
\end{array}\right)\right\|}{\|e\|} \\
& =\sqrt{\frac{\left(e_{1} e_{2}-e_{c} e_{3}\right)^{2}+\left(e_{1} e_{3}\right)^{2}}{e_{1}^{2}+e_{2}^{2}+e_{3}^{2}+e_{c}^{2}}} \\
& \leq \sqrt{\frac{\left(\left|e_{1}\right|\left|e_{2}\right|+\left|e_{c}\right|\left|e_{3}\right|\right)^{2}+\left(e_{1} e_{3}\right)^{2}}{e_{1}^{2}+e_{2}^{2}+e_{3}^{2}+e_{c}^{2}}} \\
& =\left(\frac{2\left|e_{1}\right|\left|e_{2}\right|\left|e_{3}\right|\left|e_{c}\right|}{e_{1}^{2}+e_{2}^{2}+e_{3}^{2}+e_{c}^{2}}+\frac{\left(e_{c} e_{3}\right)^{2}}{e_{1}^{2}+e_{2}^{2}+e_{3}^{2}+e_{c}^{2}}\right. \\
& \left.+\frac{\left(e_{1} e_{2}\right)^{2}+\left(e_{1} e_{3}\right)^{2}}{e_{1}^{2}+e_{2}^{2}+e_{3}^{2}+e_{c}^{2}}\right)^{1 / 2} \\
& \leq \sqrt{\frac{2\left|e_{1}\right|\left|e_{2}\right|\left|e_{3}\right|\left|e_{c}\right|}{e_{1}^{2}+e_{2}^{2}+e_{3}^{2}+e_{c}^{2}}+\frac{\left(e_{c} e_{3}\right)^{2}}{e_{c}^{2}}+\frac{\left(e_{1} e_{2}\right)^{2}+\left(e_{1} e_{3}\right)^{2}}{e_{1}^{2}}} \\
& =\sqrt{\frac{2\left|e_{1}\right|\left|e_{2}\right|\left|e_{3}\right|\left|e_{c}\right|}{e_{1}^{2}+e_{2}^{2}+e_{3}^{2}+e_{c}^{2}}+2 e_{3}^{2}+e_{2}^{2}} \\
& \leq \sqrt{\frac{2\left|e_{1}\right|\left|e_{2}\right|\left|e_{3}\right|\left|e_{c}\right|}{2\left|e_{1}\right|\left|e_{2}\right|+2\left|e_{3}\right|\left|e_{c}\right|}+2 e_{3}^{2}+e_{2}^{2}} \\
& =\sqrt{\left(\frac{1}{\left|e_{3}\right|\left|e_{c}\right|}+\frac{1}{\left|e_{1}\right|\left|e_{2}\right|}\right)^{-1}+2 e_{3}^{2}+e_{2}^{2}} \\
& \lim _{e \rightarrow 0} \frac{\left\|\left(\begin{array}{c}
n_{n p}(e, x) \\
0
\end{array}\right)\right\|}{\|e\|} \\
& \leq \lim _{e \rightarrow 0} \sqrt{\left(\frac{1}{\left|e_{3}\right|\left|e_{c}\right|}+\frac{1}{\left|e_{1}\right|\left|e_{2}\right|}\right)^{-1}+2 e_{3}^{2}+e_{2}^{2}}=0, \\
& \left.\left(\begin{array}{c}
n_{n p}(e, x) \\
0
\end{array}\right)\right|_{e=0}=0 .
\end{aligned}
$$

Therefore, the first condition in the above-mentioned theorem holds.

Now, choose suitable real constant matrix $\Omega \in R^{1 \times n}$ and $F \in R^{n \times(n+1)}$ such that

$$
\begin{gathered}
\operatorname{Re}\left[\lambda\left(\begin{array}{c}
L+F \\
\Omega
\end{array}\right)\right]<0, \\
-\max \left[\operatorname{Re} \lambda\left(\begin{array}{c}
L+F \\
\Omega
\end{array}\right)\right]>[\Gamma(q)]^{1 / q} .
\end{gathered}
$$

So, the second condition in the above-mentioned theorem holds. According to the theoremin Section 2, the adaptive synchronization between drive system (29) and response system (31) with parameter update law (32) can be achieved. 

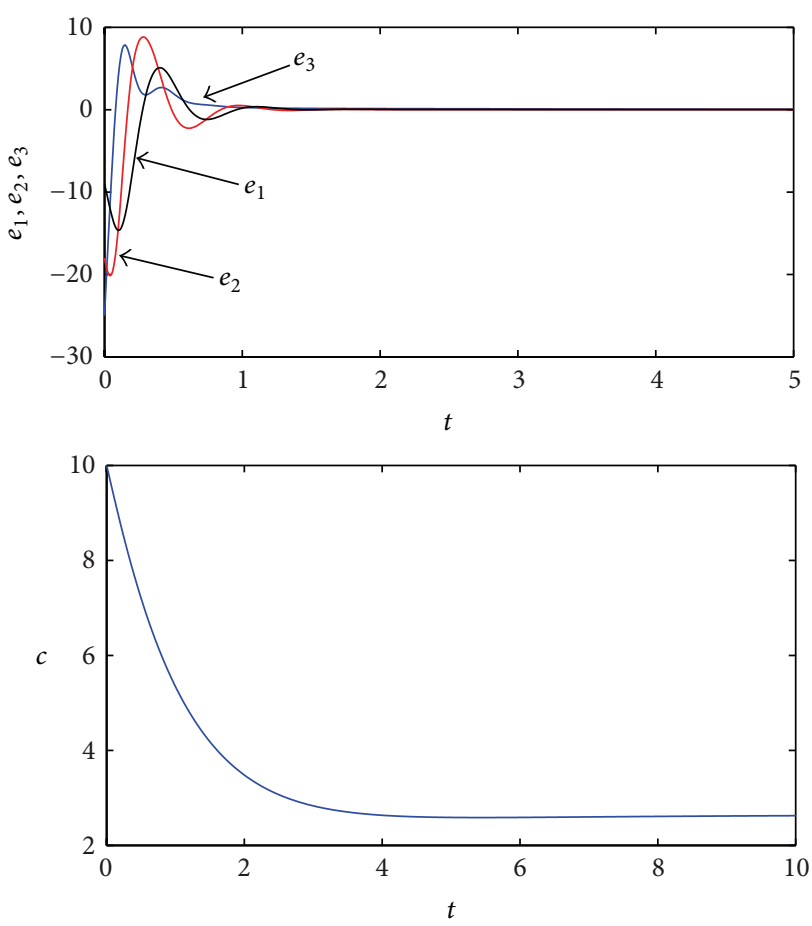

FIGURE 4: Simulation results of the adaptive synchronization for the fractional-order Lorenz chaotic system.

For example, let $F=\left(\begin{array}{cccc}0 & 0 & 0 & 0 \\ -38 & 0 & 0 & 0 \\ 0 & 0 & -1 & 0\end{array}\right)$ and $\Omega=\left(\begin{array}{llll}0 & 0 & 0 & -1\end{array}\right)$. So $\left(\begin{array}{c}L+F \\ \Omega\end{array}\right)=\left(\begin{array}{cccc}-10 & 10 & 0 & 0 \\ -10 & -1 & 0 & 0 \\ 0 & 0 & -1 & 0 \\ 0 & 0 & 0 & -1\end{array}\right)$. Therefore, $\lambda_{i}=-1(i=1,2)$, $\lambda_{ \pm}=-5.5 \pm 8.9303 j$, and $-\max \left[\operatorname{Re} \lambda\left(\begin{array}{c}L+F \\ \Omega\end{array}\right)\right]=1>$ $[\Gamma(q)]^{1 / q}=0.9722$, respectively. Simulation results are shown in Figure 4. Here, $c(0)=10$.

\section{Conclusions}

One adaptive synchronization scheme for a class of fractional-order chaotic system with fractional-order $1<q<2$ is suggested in this paper. This synchronization approach is based on a new stability result of equilibrium point in nonlinear fractional-order systems for fractionalorder lying in $1<q<2$. In order to verify the effectiveness of the adaptive synchronization approach, the adaptive synchronization for the fractional-order Lorenz chaotic system with fractional-order $1<q<2$ is considered. Numerical simulations show the validity and feasibility of the proposed scheme. The current results in this paper can be extended to several unknown parameters of the fractionalorder chaotic systems. Moreover, this synchronization approach can be applied to other fractional-order chaotic systems.

\section{Conflict of Interests}

The authors declare that there is no conflict of interests regarding the publication of this paper.

\section{References}

[1] L. Chen, Y. He, Y. Chai, and R. Wu, "New results on stability and stabilization of a class of nonlinear fractional-order systems," Nonlinear Dynamics, vol. 75, no. 4, pp. 633-641, 2014.

[2] R. Hilfer, Applications of Fractional Calculus in Physics, World Scientific, Singapore, 2000.

[3] M. Naber, "Time fractional Schrödinger equation," Journal of Mathematical Physics, vol. 45, no. 8, pp. 3339-3352, 2004.

[4] I. Grigorenko and E. Grigorenko, "Chaotic dynamics of the fractional Lorenz system," Physical Review Letters, vol. 91, Article ID 034101, 2003.

[5] P. Zhou, R. Ding, and Y. X. Cao, "Multi drive-one response synchronization for fractional-order chaotic systems," Nonlinear Dynamics, vol. 70, no. 2, pp. 1263-1271, 2012.

[6] Z. M. Ge and C. Y. Ou, "Chaos in a fractional order modified Duffing system," Chaos, Solitons and Fractals, vol. 34, no. 2, pp. 262-291, 2007.

[7] D. Cafagna and G. Grassi, "Hyperchaos in the fractional-order Ro $\mu$ ssler system with lowest-order," International Journal of Bifurcation and Chaos, vol. 19, no. 1, pp. 339-347, 2009.

[8] P. Zhou and F. Yang, "Hyperchaos, chaos, and horseshoe in a 4D nonlinear system with an infinite number of equilibrium points," Nonlinear Dynamics, vol. 76, no. 1, pp. 473-480, 2014.

[9] J. C. Sprott, Chaos and Time-Series Analysis, Oxford University Press, Oxford, UK, 2003.

[10] G. R. Chen and X. Dong, "From chaos to order perspectives," in Methodologies and Applications, World scientific, Singapore, 1998.

[11] X. Y. Wang and J. M. Song, "Synchronization of the fractional order hyperchaos Lorenz systems with activation feedback control," Communications in Nonlinear Science and Numerical Simulation, vol. 14, no. 8, pp. 3351-3357, 2009.

[12] P. Zhou and W. Zhu, "Function projective synchronization for fractional-order chaotic systems," Nonlinear Analysis: Real World Applications, vol. 12, no. 2, pp. 811-816, 2011.

[13] R. Zhang and S. Yang, "Adaptive synchronization of fractionalorder chaotic systems via a single driving variable," Nonlinear Dynamics, vol. 66, no. 4, pp. 831-837, 2011.

[14] L. L. Li and J. D. Cao, "Cluster synchronization in an array of coupled stochastic delayed neural networks via pinning control," Neurocomputing, vol. 74, no. 5, pp. 846-856, 2011.

[15] J. Q. Lu and J. D. Cao, "Adaptive synchronization in tree-like dynamical networks," Nonlinear Analysis: Real World Applications, vol. 8, no. 4, pp. 1252-1260, 2007.

[16] J. Q. Lu and J. D. Cao, "Adaptive synchronization of uncertain dynamical networks with delayed coupling," Nonlinear Dynamics, vol. 53, no. 1-2, pp. 107-115, 2008.

[17] F. Mainardi, "The fundamental solutions for the fractional diffusion-wave equation," Applied Mathematics Letters, vol. 9, no. 6, pp. 23-28, 1996.

[18] L. Beghin and E. Orsingher, "The telegraph process stopped at stable-distributed times and its connection with the fractional telegraph equation," Fractional Calculus \& Applied Analysis, vol. 6, no. 2, pp. 187-204, 2003.

[19] W. Zhang, X. Cai, and S. Holm, "Time-fractional heat equations and negative absolute temperatures," Computers \& Mathematics with Applications, vol. 67, no. 1, pp. 164-171, 2014. 


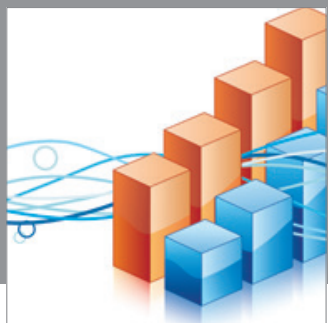

Advances in

Operations Research

mansans

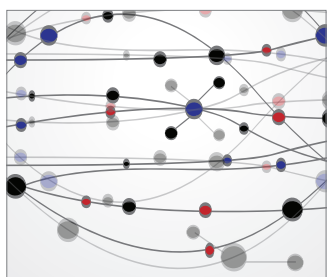

The Scientific World Journal
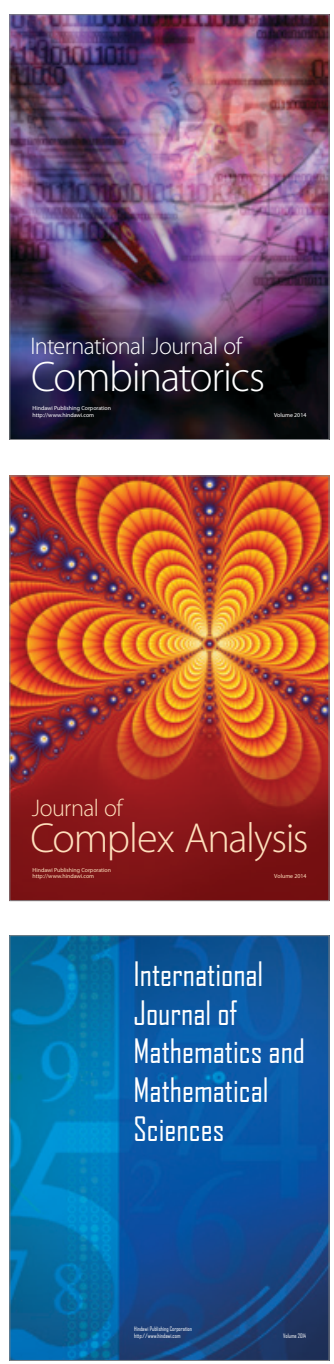
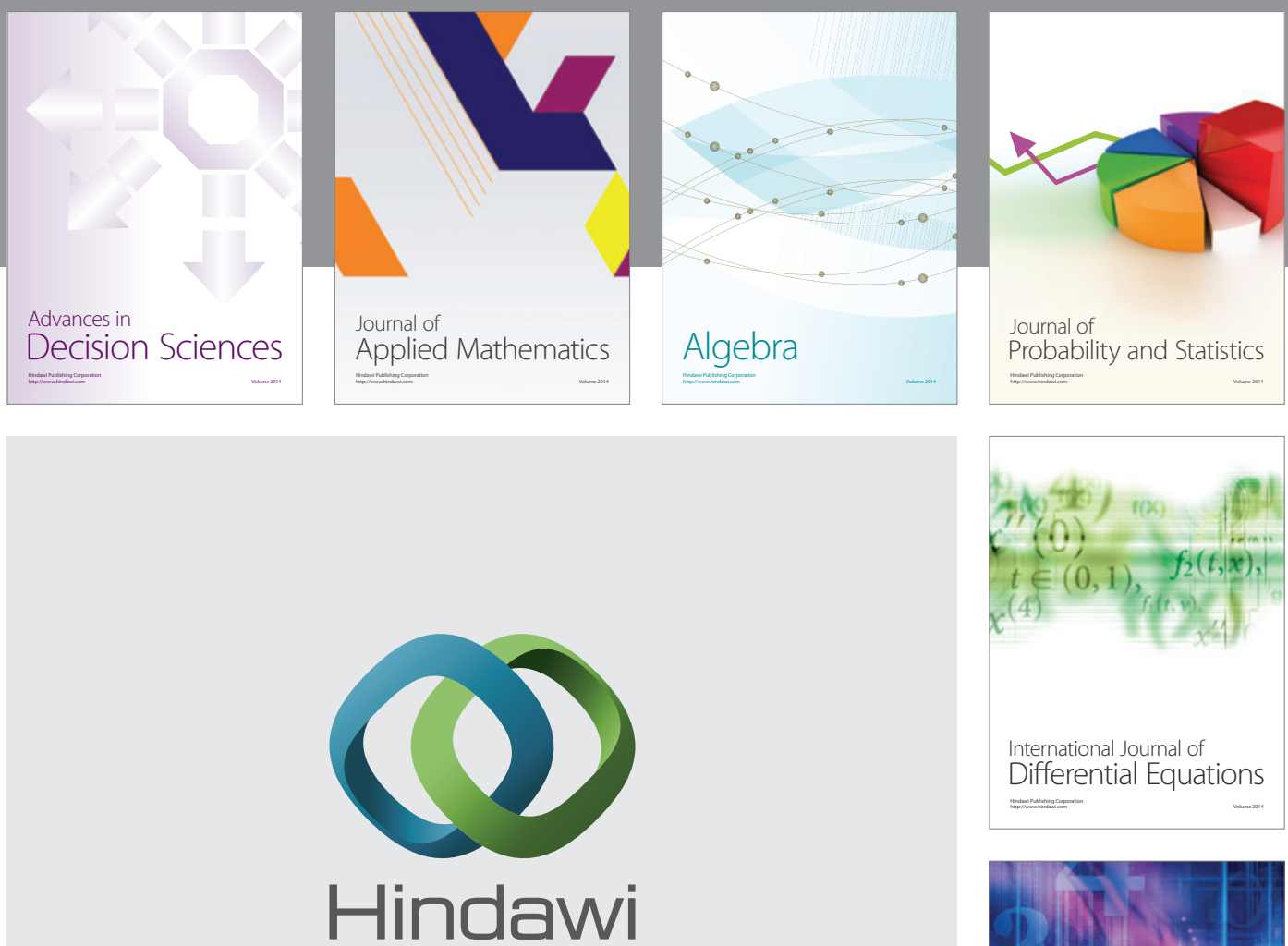

Submit your manuscripts at http://www.hindawi.com
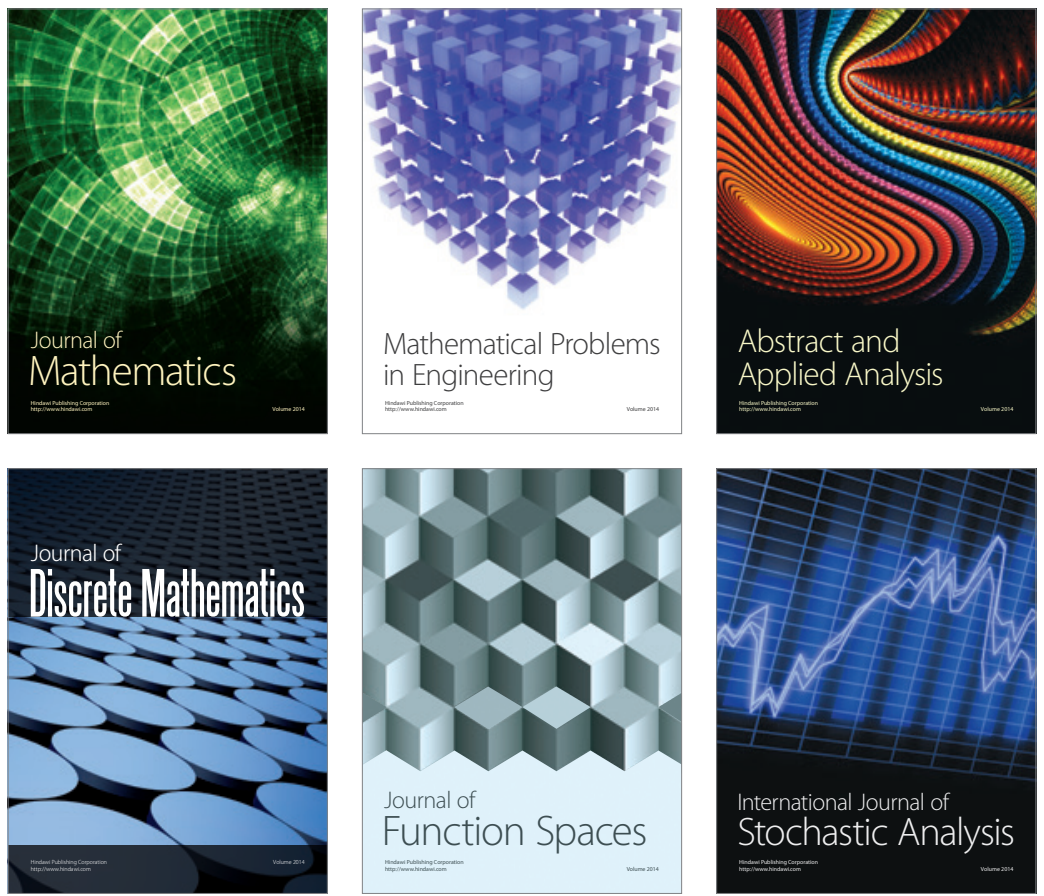

Journal of

Function Spaces

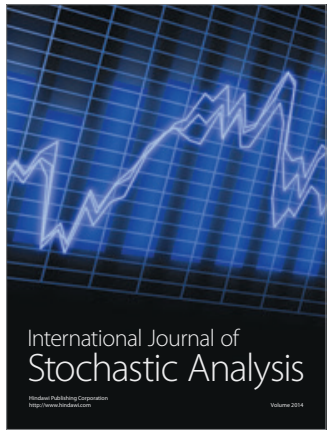

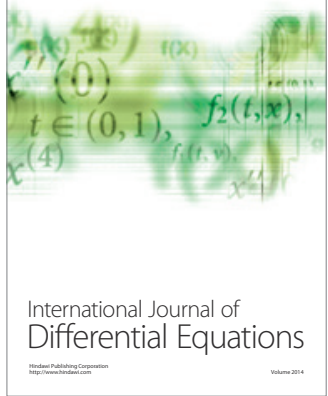
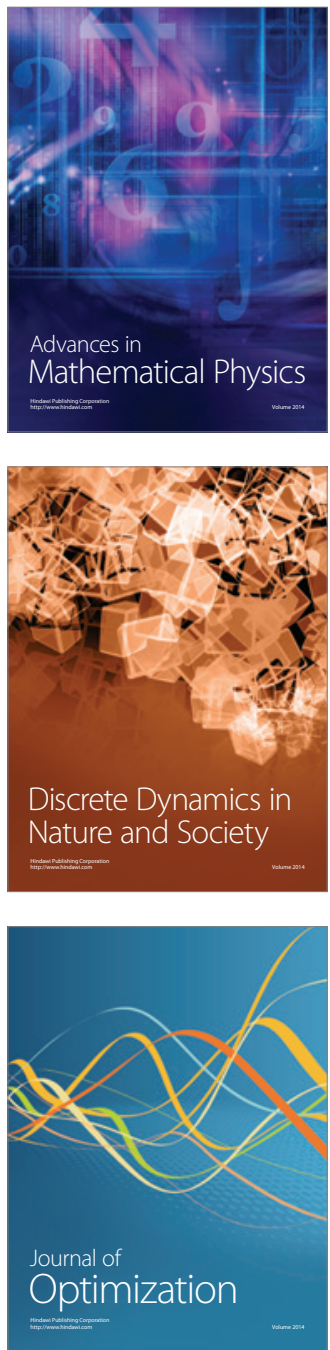